\title{
4D Laboratory X-ray Microscopy for the in-Situ Investigation of Drug Release in a Push-pull Osmotic Pump Tablet
}

\author{
Hrishikesh Bale $^{1}$, Will Harris ${ }^{1}$ and Arno Merkle ${ }^{1}$ \\ ${ }^{1 .}$ Carl Zeiss X-Ray Microscopy, Inc., Pleasanton, CA, USA
}

Depending on the specific treatment of disease or underlying condition, modern oral drugs are engineered to deliver the dosage, either as immediate-release, delayed release, prolonged release or in some instances targeted release. In contrast to the immediate-release concept of drug delivery, the latter methods require complex formulation, packaging and encapsulation of the drugs so that the initiation, release duration and release rate of the drug is achieved according to a certain designed delivery profile.[1] Osmotic controlled release system is an advanced approach which utilizes a semipermeable cellulose or polymer based membrane, enclosing single or multiple layers of either drug or combination of drug and push layers. The membrane is designed to allow only the inward permeation of water as the tablet enters the gastrointestinal (GI) tract and restricts diffusion of the drug out of the casing, except through a laser drilled orifice. The water that diffuses via osmosis, dissolves the active drug and builds up internal pressure causing the release of the drug from a laser drilled orifice. The osmotic release mechanism has many inherent advantages over other methods, in that, it is less influenced by factors such as motility, differing $\mathrm{pH}$ environments in the GI tract, allows tuning the drug delivery rates and is most effective for delivering poorly soluble drugs. However, due to their relatively higher complexity, they are much more challenging to develop and manufacture. The design for accurate and effective drug delivery of a particular drug chemistry utilizes theoretical models that compute the osmotic pressure, diffusivity, flow rate and timing of delivery. These calculations require input of experimentally determined parameters using imaging techniques such as light microscopy and scanning electron microscopy (SEM). However, observation of the behavior of the drug over time can only be achieved through interrupted experiments requiring destructive sectioning of the samples. Moreover, the optical microscopy and SEM are surface imaging methods and do not reveal sub-surface information.

$\mathrm{X}$-ray microscopy is a powerful imaging technique, that enables the full 3D non-destructive investigation of samples. Its non-invasive nature, permits visualizing internal microstructure, voids and particle distribution, including processing and manufacturing defects. Besides, it also enables four-dimensional (4D) in-situ observations of time dependent changes in a sample subject to a simulated environment. We present here, results from a 4D in-situ, non-destructive investigation performed on a model pharmaceutical tablet based on push-pull osmotic pump mechanism. The tablet was exposed to water, inside a customized in-situ stage simulating its operation in the GI tract. Employing X-ray microscopy (XRM) in a computed tomography (Micro-CT) approach we performed 3D imaging at $4 \mu \mathrm{m} /$ voxel resolution at several time points over 18 hours, capturing the changes in microstructure as water diffuses through the membrane and activates drug delivery. The imaging clearly resolved the osmotic membrane, the bilayer composition of the tablet and the laser drilled orifice with a high contrast. Furthermore, as water diffuses into the tablet, the dissolution and movement of push layer particles within the drug layer along with the gradual drug delivery was captured in the tomography images. Through the 3D reconstructed virtual cross-sectional images, a quantitative analysis of the dissolution rate and measurements of the tablet swelling were carried out. The results clearly highlight the advantages of using X-ray microscopy for visualizing the progression of drug release, which may be used for further modeling and simulation studies in designing newer, more accurate and efficient drug release mechanisms. 


\section{References:}

[1] Dwarakanadha, R., Swarnalatha, D., Int. J. Pharm. Technol. Res. 2 (2010), p. 2025.

[2] Malaterre V et al, 2009. Int J Pharm. 376 (1-2) (2009), p. 56.
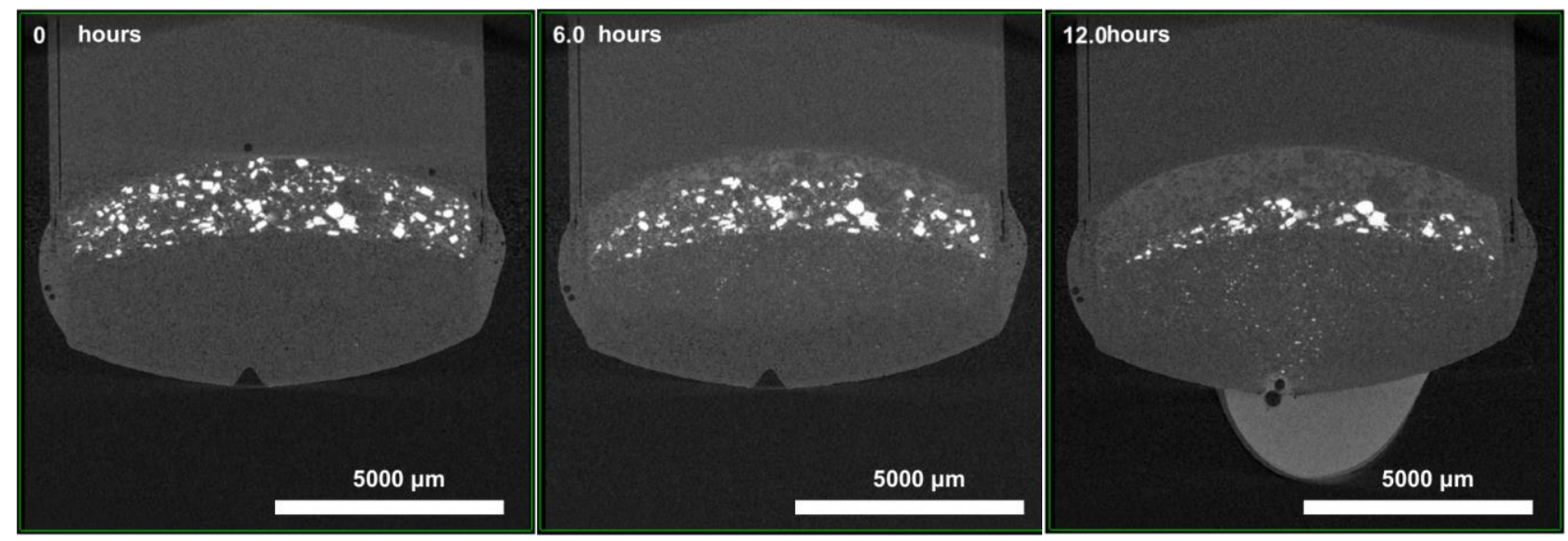

Figure 1. Central cross-sectional slices from in-situ tomography data collected at 0mins, 6 hours and 12 hour duration from the moment the tablet was immersed in water.

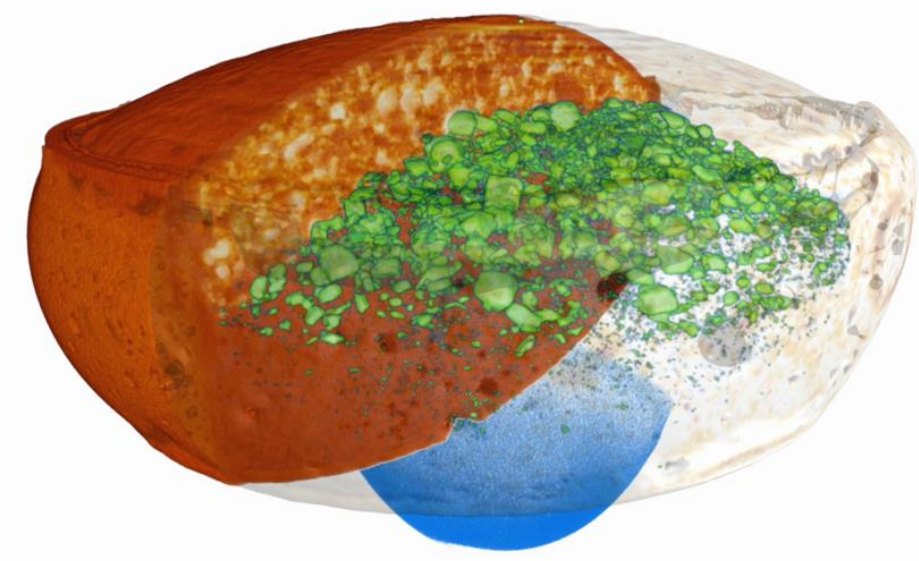

Figure 2. 3D rendering of the tablet after 10 hours of immersion in water. False colors were assigned in the 3D rendering showing push layer particles (green) and the released drug(blue). 